Research Article

\title{
Clinical Analysis of Cervical Lymph Node Metastasis Risk Factors and the Feasibility of Prophylactic Central Lymph Node Dissection in Papillary Thyroid Carcinoma
}

\author{
Yifan Chen, ${ }^{1,2}$ Shuo Chen, ${ }^{2,3}$ Xiaoying Lin,,2 Xiangqing Huang, ${ }^{1,2}$ Xiaofang Yu, ${ }^{1,4}$ \\ and Juying Chen $\mathbb{1}^{1,2}$ \\ ${ }^{1}$ Department of General Surgery, South Branch of Fujian Provincial Hospital, Fuzhou 350000, Fujian, China \\ ${ }^{2}$ Provincial Clinical Medical College of Fujian Medical University, Fuzhou 350000, Fujian, China \\ ${ }^{3}$ Department of Gynaecology and Obstetrics, South Branch of Fujian Provincial Hospital, Fuzhou 350000, Fujian, China \\ ${ }^{4}$ Department of Anesthesiology, South Branch of Fujian Provincial Hospital, Fuzhou 350000, Fujian, China
}

Correspondence should be addressed to Juying Chen; demoncyf@126.com

Received 18 October 2020; Revised 4 January 2021; Accepted 17 January 2021; Published 31 January 2021

Academic Editor: Claudio Casella

Copyright (c) 2021 Yifan Chen et al. This is an open access article distributed under the Creative Commons Attribution License, which permits unrestricted use, distribution, and reproduction in any medium, provided the original work is properly cited.

Objective. To identify the risk factors for cervical lymph node metastasis (CLNM) and the feasibility of prophylactic central lymph node dissection. Methods. The characteristics of 1107 patients were extracted and analyzed. Univariate and multivariate analyses were used to identify risk factors associated with lymph node metastasis. The relationship between the central lymph node dissection (CLND) and lateral lymph node metastasis (LLNM) was analyzed using the correlation analysis. Results. The probability of CLNM was closely related to the male gender, age $<55$, and the increase of tumor size. Those patients with an increase in tumor size and CLNM were extremely prone to LLNM. Also, LLNM was more likely to happen in those with the more positive central lymph nodes. Routine prophylactic central lymph node dissection (P-CLND) did not increase the risk of complications. Conclusion. P-CLND should be considered as a reasonable surgical treatment for PTC.

\section{Introduction}

Following the improvements of ultrasonography (US) and US-guided fine-needle aspiration biopsy (FNAB), the incidence of thyroid carcinoma has been increasing over recent decades. Papillary thyroid carcinoma (PTC) is the most common thyroid malignancy. As an indolent disease, the majority of patients with PTC have an excellent 10-year prognosis with a survival rate of more than $99 \%$ [1]. The favorable prognosis results in controversy regarding the optimal therapeutic strategy for PTC. Some researchers, such as Ito, propose close follow-up for PTC patients instead of surgery [2]. Sugitani believes that delayed surgery has no effect on the outcome [3]. However, according to other studies, lymph node metastasis (LNM), especially cervical lymph node metastasis (CLNM), occurs in over $50 \%$ of patients with PTC $[4,5]$. It is possible that besides being an independent risk factor for lateral lymph node metastasis (LLNM) [6-9], CLNM also elevates the recurrence rate and disease-specific mortality [10-12]. Repeated surgery may lead to an increase of complications, such as damage to the recurrent laryngeal nerve and hypoparathyroidism, which affect the life quality of patients. Central lymph node dissection (CLND) is recommended for patients who are suspected of CLNM in preoperative assessment. Yet, prophylactic central lymph node dissection (P-CLND) for patients with clinically node-negative is still controversial.

In the present study, we performed a retrospective analysis that focused on PTC patients without clinical evidence for CLNM, who received P-CLND. The aim of the study was to analyze the incidence, pattern, and risk factors of lymph node metastasis in PTC patients, to identify the high-risk patients and evaluate postoperative complications 
of P-CLND, so as to provide new evidence for the treatment of PTC patients.

\section{Materials and Methods}

2.1. Patients. A total of 1107 PTC patients who underwent thyroidectomy and P-CLND with or without lateral lymph node dissection between January 2015 and December 2019 at the Department of General Surgery, Fujian Provincial Hospital, were included in the study. Patients were enrolled according to the following criteria: (1) all cases that were preoperatively suspected as PTC following US examination, part of which were confirmed by FNAB-US characteristics include ultrasound intensity, composition, echoic distribution, tumor border, shape, calcification, aspect ratio, and blood flow; neck/thorax computer tomography (CT) is not systematically performed in our hospital-(2) all patients without clinical evidence of CLNM, who underwent unilateral or bilateral thyroidectomy with P-CLND and with or without lateral lymph node dissection; (3) all patients who were proven to have PTC by intraoperative frozen and postoperative pathological examination (all specimens were independently examined by two pathologists); and (4) all patients who were without a history of previous thyroid or neck surgery.

The study was approved by the Institutional Review Board of Fujian Provincial Hospital.

2.2. Treatment. In our hospital, P-CLND is routinely performed for PTC patients. Ipsilateral lobe and isthmus resection was performed for unilateral primary lesions. Total thyroidectomy was performed for bilateral primary lesions, and unilateral primary lesions requiring iodine-131 treatment. Lateral lymph node dissection is performed only if there is radiographic, cytopathologic, or intraoperative frozen pathological evidence suggestive of lymph node metastasis. Central lymph nodes include pretracheal, paratracheal, and prelaryngeal lymph nodes and lymph nodes located along the recurrent laryngeal nerve. Lateral lymph nodes include levels I, II, III, IV, and V. Levels II, III, and IV are routinely dissected. Levels I and V are dissected when lymph nodes metastases are suspected or confirmed. All patients received thyroid-stimulating hormone (TSH) suppression with levothyroxine (L-T4) after surgery. Part of the patients underwent iodine-131 treatment.

2.2.1. Assessment of Surgical Complications. Serum parathyroid hormone (PTH) and total serum calcium were measured on the first postoperative day and every week for 3 consecutive weeks thereafter. Hypoparathyroidism (hypoPTH) was considered permanent when total serum calcium and PTH were still under normal value, or symptoms still existed at 6 months after surgery. Direct fiber-optic laryngoscopy was performed in all patients before and after surgery, to assess cord motility. Recurrent laryngeal nerve injury was considered permanent when the cord palsy persisted 6 months after surgery.
2.2.2. Statistical Analysis. All statistical analyses were performed using the Statistical Package for Social Sciences (SPSS, Inc., Chicago, IL, USA). Univariate and multivariate analyses were performed to determine the significance of clinical characteristics. The univariate analyses were performed using the Chi-square test and Fisher's exact test. Variables with $P<0.05$ in the univariate analysis were included in the multivariate analysis. The multivariate analyses were performed using binary logistic regression analysis. The results are presented as odds ratios (OR) with 95\% confidence intervals $(\mathrm{CI})$ and $P$ values. A $P$ value of $<0.05$ was considered statistically significant.

\section{Results}

There were 1107 patients enrolled in this study, including 828 females (74.8\%) and 279 males (25.2\%). In most of them, PTC was incidentally found during regular medical examinations. Others had symptoms, such as anterior neck mass, voice change, and lateral neck mass. The age of patients at the diagnosis time ranged from 15 to 80 years. Diameter ranged from 0.1 to $5.0 \mathrm{~cm}$. 384 patients had a tumor size $\leq 5 \mathrm{~mm}(34.7 \%), 366$ patients had a tumor size $>5 \mathrm{~mm}$ but $\leq 10 \mathrm{~mm}$ (33.1\%), 294 patients had a tumor size $>10 \mathrm{~mm}$ but $\leq 20 \mathrm{~mm}(26.6 \%)$, and 63 patients had a tumor size $>20 \mathrm{~mm}$ (5.6\%). Among all patients, 225 patients exhibited multifocality in one thyroid lobe, and 171 patients had bilaterality. A total of 120 patients had adjacent structure invasion, such as strap muscles, recurrent laryngeal nerve. A total of 195 patients had Hashimoto thyroiditis.

After postoperative pathological examinations, LNM was present in 474 patients. Among them, 453 patients had CLNM (95.6\%), 117 patients had LLNM (24.7\%), and 21 patients had lateral without central lymph node metastasis. In this study, all patients received P-CLND. Seven hundred eighty-nine patients underwent lobectomy plus ipsilateral CLND, 174 patients underwent total thyroidectomy plus bilateral CLND, and 144 patients underwent total thyroidectomy plus bilateral CLND and unilateral LLND. The patients' characteristics are summarized in Table 1.

Table 2 shows the postoperative complications. Seventysix patients had hypoparathyroidism that resolved within 3-6 months in 67 patients $(6 \%)$ and was persistent in 9 patients $(2.8 \%)$. Transient hypoparathyroidism was observed in 60 patients undergoing total thyroidectomy and in seven patients having lobectomy. Persistent hypoparathyroidism was observed in all patients who underwent total thyroidectomy. Among 34 cases with vocal cord palsy, which implied recurrent laryngeal nerve injury, 22 (2\%) recovered within 1 to 6 months while the other $12(1.1 \%)$ patients had a persistent injury. Two patients $(0.2 \%)$ experienced bleeding problems after surgery, which led to reoperation. Chyle leakage, which was cured within 20 days, was observed in 5 patients $(3.5 \%)$ undergoing total thyroidectomy plus CLND and LLND. None of the patients in this study had hematoma, tracheal leak, Horner's syndrome, and other complications.

We first analyzed the relationship between clinical characteristics and LNM. Univariate analysis revealed that 
TABLE 1: Clinicopathologic characteristics of patients.

\begin{tabular}{lc}
\hline Characteristics & Number \\
\hline $\begin{array}{l}\text { Number of patients } \\
\text { Age at diagnosis (years) }\end{array}$ & 1107 \\
$\quad<55$ & 816 \\
$\geq 55$ & 291 \\
\hline Gender & \\
Female & 828 \\
$\quad$ Male & 279 \\
\hline Tumor size (mm) & \\
$\quad \leq 5$ & 384 \\
$>5, \leq 10$ & 366 \\
$>10, \leq 20$ & 294 \\
$>20$ & 63 \\
\hline Multifocal neoplasms & 225 \\
Bilateral neoplasms & 171 \\
Extrathyroidal extension & 120 \\
Hashimoto thyroiditis & 195 \\
Lymph node metastasis & 474 \\
Central lymph node metastasis & 453 \\
Lateral lymph node metastasis & 117 \\
\hline Surgery procedure & 789 \\
Lobectomy plus ipsilateral CLND & 174 \\
Total thyroidectomy plus bilateral CLND & 144 \\
Total thyroidectomy plus bilateral CLND and & \\
unilateral LLND & \\
\hline
\end{tabular}

CLND: central lymph node dissection; LLND: lateral lymph node dissection.

TABle 2: Postoperative complications.

\begin{tabular}{lcc}
\hline \multirow{2}{*}{ Complications } & \multicolumn{2}{c}{ Number (\%) } \\
& Transient & Persistent \\
\hline Hypoparathyroidism & $67 / 1107(6 \%)$ & $9 / 318(2.8 \%)$ \\
Lobectomy & $7 / 789(0.9 \%)$ & 0 \\
Total thyroidectomy & $60 / 318(18.9 \%)$ & $9 / 318(2.8 \%)$ \\
Recurrent laryngeal nerve injury & $22 / 1107(2 \%)$ & $12 / 1107(1.1 \%)$ \\
Chyle leakage & $5 / 144(3.5 \%)$ & 0 \\
Bleeding & $2 / 1107(0.2 \%)$ & 0 \\
Hematoma & 0 & 0 \\
Tracheal leak & 0 & 0 \\
Horner's syndrome & 0 & 0 \\
\hline
\end{tabular}

male gender, age $<55$, the increase in tumor size, multifocality, bilaterality, and extrathyroidal extension were significant predictors, but not Hashimoto thyroiditis. Then, multivariate logistic regression analysis was used to confirm the independently predictive factors of LNM. As shown in Table 3 , the male gender, age $<55$, the increase in tumor size, and multifocality still resulted as independent factors. In the univariate analysis, CLNM was significantly associated with male gender, age $<55$, the increase in tumor size, multifocality, and extrathyroidal extension. Nevertheless, bilaterality and Hashimoto thyroiditis were irrelevant to CLNM. For multivariate analysis, male gender, age $<55$, and the increase in tumor size resulted as independent predictive factors for CLNM. The results are shown in Table 4. Univariate analysis showed a statistically significant association between LLNM and the increase in tumor size, extrathyroidal extension, and CLNM. In contrast, no significant association was found between LLNM and gender, age, multifocality, and bilaterality. In the multivariate analysis, LLNM was associated with an increase in tumor size and CLNM (Table 5). According to the previous studies, approximately $80 \%$ of LLNM was found in patients with $\geq 2$ positive central lymph nodes [13-15]. We tested this hypothesis in the present study, and our results revealed that the number of positive central lymph nodes had a positive correlation with the incidence of LLNM (results are shown in Figures 1 and 2).

\section{Discussion}

The incidence of thyroid cancer has been increasing faster than any other cancer over recent years, especially in developed nations, where people have high access to healthcare [16]. PTC, the most common thyroid malignancy, is a lymphotropic tumor that frequently presents with neck node metastases [17]. More than $99 \%$ of PTC patients have a 10year-survival rate [1]. Nevertheless, some patients suffer from recurrence, reoperation, and disease-specific mortality.

CLNM is common in PTC, with an occurrence rate that ranges from $18 \%$ to $90 \%[4,5,14,18,19]$. We focused on the association of LNM with the clinicopathological characteristics in this study. The male gender, age $<55$, and the increase in tumor size were closely related to the probability of LNM and CLNM. This finding is consistent with the data reported [20-23]. Those patients with an increase in tumor size and CLNM were extremely prone to LLNM. Interestingly, we found multifocality only was an independent risk factor for LNM, rather than for CLNM and LLNM. This finding is inconsistent with previous studies [23-26]. Therefore, for male and younger patients, we should pay more attention to LNM, especially CLNM. For patients with larger tumor size, we should pay attention not only to CLNM but also to LLNM.

In this study, we found CLNM was an independent risk factor for LLNM, and the rising number of positive central lymph nodes increased the incidence of LLNM. The relationship between positive central lymph nodes and LLNM may be explained by the dissemination of PTC through the lymphatic system $[27,28]$. Lymph node metastases tend to occur in consecutive orders from the central compartment, followed by the lateral compartment and then mediastinal lymph nodes [6,29]. Metastases in the lateral compartment without positive central lymph nodes are very rare [30]. In our study, only 21 patients had skip metastasis. Central lymph node dissection is seen as an effective approach to cut off lymphatic flow to lateral lymph nodes [31]. Some studies have shown that their surgical removal of lymph node metastases improves survival rates [32, 33].

The US is regarded as a routine method to evaluate metastasis in lymph nodes. However, the sensitivity of the US in the evaluation of CLNM is poor, and lymph node metastases of level VI are not detectable preoperatively $[34,35]$. In our study, 453 patients $(40.9 \%)$ without evidence of CLNM following the US examination had cervical lymphatic invasion, which was confirmed by postoperation 
TABLE 3: Univariable and multivariate analysis for LNM.

\begin{tabular}{|c|c|c|c|c|c|}
\hline \multirow[b]{2}{*}{ Risk factors } & \multicolumn{3}{|c|}{ Univariate analysis } & \multicolumn{2}{|c|}{ Multivariate analysis } \\
\hline & LNM+ & LNM- & $P$ value & $P$ value & OR $(95 \%$ CI $)$ \\
\hline \multicolumn{6}{|l|}{ Age at diagnosis (years) } \\
\hline$<55$ & 387 & 429 & $<0.001$ & $<0.001$ & $2.128(1.563-2.897)$ \\
\hline$\geq 55$ & 87 & 204 & & & \\
\hline \multicolumn{6}{|l|}{ Gender } \\
\hline Female & 333 & 495 & 0.003 & $<0.001$ & $0.57(0.421-0.773)$ \\
\hline Male & 141 & 138 & & & \\
\hline \multicolumn{6}{|l|}{ Tumor size (mm) } \\
\hline$\leq 5$ & 114 & 270 & & $<0.001$ & \\
\hline$>5, \leq 10$ & 135 & 231 & $<0.001$ & $<0.001$ & $7.252(3.652-14.399)$ \\
\hline$>10, \leq 20$ & 174 & 120 & & $<0.001$ & $6.526(3.301-12.903)$ \\
\hline$>20$ & 51 & 12 & & 0.002 & $3.052(1.527-6.101)$ \\
\hline Multifocal neoplasms & & & $<0.001$ & 0.001 & $1.712(1.273-2.370)$ \\
\hline Positive & 123 & 102 & & & \\
\hline Negative & 351 & 531 & & & \\
\hline Bilateral neoplasms & & & 0.005 & 0.754 & \\
\hline Positive & 90 & 81 & & & \\
\hline Negative & 384 & 552 & & & \\
\hline Extrathyroidal extension & & & 0.001 & 0.442 & \\
\hline Positive & 69 & 51 & & & \\
\hline Negative & 405 & 582 & & & \\
\hline Hashimoto thyroiditis & & & 0.130 & & \\
\hline Positive & 93 & 102 & & & \\
\hline Negative & 381 & 531 & & & \\
\hline
\end{tabular}

LNM: lymph node metastasis.

TABLE 4: Univariable and multivariate analysis for CLNM.

\begin{tabular}{|c|c|c|c|c|c|}
\hline \multirow[b]{2}{*}{ Risk factors } & \multicolumn{3}{|c|}{ Univariate analysis } & \multicolumn{2}{|c|}{ Multivariate analysis } \\
\hline & CLNM+ & CLNM- & $P$ value & $P$ value & OR $(95 \% \mathrm{CI})$ \\
\hline Age at diagnosis (years) & & & $<0.001$ & $<0.001$ & $2.187(1.606-2.978)$ \\
\hline$<55$ & 372 & 444 & & & \\
\hline$\geq 55$ & 81 & 210 & & & \\
\hline Gender & & & 0.012 & 0.005 & $0.651(0.484-0.876)$ \\
\hline Female & 321 & 507 & & & \\
\hline Male & 132 & 147 & & & \\
\hline Tumor size (mm) & & & $<0.001$ & & \\
\hline$\leq 5$ & 108 & 276 & & $<0.001$ & \\
\hline$>5, \leq 10$ & 135 & 231 & & $<0.001$ & $3.415(1.896-6.150)$ \\
\hline$>10, \leq 20$ & 168 & 126 & & $<0.001$ & $2.887(1.614-5.162)$ \\
\hline$>20$ & 42 & 21 & & 0.261 & $1.403(0.777-2.533)$ \\
\hline Multifocal neoplasms & & & 0.004 & 0.600 & \\
\hline Positive & 111 & 114 & & & \\
\hline Negative & 342 & 540 & & & \\
\hline Bilateral neoplasms & & & 0.062 & & \\
\hline Positive & 81 & 90 & & & \\
\hline Negative & 372 & 564 & & & \\
\hline Extrathyroidal extension & & & $<0.001$ & 0.617 & \\
\hline Positive & 66 & 54 & & & \\
\hline Negative & 387 & 600 & & & \\
\hline Hashimoto thyroiditis & & & 0.247 & & \\
\hline Positive & 87 & 108 & & & \\
\hline Negative & 366 & 546 & & & \\
\hline
\end{tabular}

CLNM: central lymph node metastasis.

histopathologic examination. In a total of 144 patients with evidence of LLNM by US examination, 117 patients $(81.3 \%)$ had lateral lymph node metastases that were also confirmed by histopathologic examination. The huge gaps that were observed between US examination forecasting and CLNM were unexpected.

On the other hand, numerous studies tried to identify what kind of PTC patients were more likely to have CLNM 
TABLE 5: Univariable and multivariate analysis for LLNM.

\begin{tabular}{|c|c|c|c|c|c|}
\hline \multirow[b]{2}{*}{ Risk factors } & \multicolumn{3}{|c|}{ Univariate analysis } & \multicolumn{2}{|c|}{ Multivariate analysis } \\
\hline & LLNM+ & LLNM- & $P$ value & $P$ value & OR $(95 \% \mathrm{CI})$ \\
\hline Age at diagnosis (years) & & & 0.134 & & \\
\hline$<55$ & 93 & 723 & & & \\
\hline$\geq 55$ & 24 & 267 & & & \\
\hline Gender & & & 0.429 & & \\
\hline Female & 84 & 744 & & & \\
\hline Male & 33 & 246 & & & \\
\hline Tumor size (mm) & & & $<0.001$ & & \\
\hline$\leq 5$ & 15 & 369 & & $<0.001$ & \\
\hline$>5, \leq 10$ & 21 & 345 & & $<0.002$ & $13.165(6.177-28.060)$ \\
\hline$>10, \leq 20$ & 51 & 243 & & $<0.003$ & $11.946(5.909-24.151)$ \\
\hline$>20$ & 30 & 33 & & $<0.004$ & $4.917(2.614-9.250)$ \\
\hline Multifocal neoplasms & & & 0.958 & & \\
\hline Positive & 24 & 201 & & & \\
\hline Negative & 93 & 789 & & & \\
\hline Bilateral neoplasms & & & 0.109 & & \\
\hline Positive & 24 & 147 & & & \\
\hline Negative & 93 & 843 & & & \\
\hline Extrathyroidal extension & & & 0.006 & 0.445 & \\
\hline Positive & 21 & 96 & & & \\
\hline Negative & 96 & 894 & & & \\
\hline Hashimoto thyroiditis & & & 0.503 & & \\
\hline Positive & 18 & 177 & & & \\
\hline Negative & 99 & 813 & & & \\
\hline CLNM & & & $<0.001$ & $<0.001$ & $5.463(3.264-9.143)$ \\
\hline Positive & 96 & 357 & & & \\
\hline Negative & 21 & 633 & & & \\
\hline
\end{tabular}

CLNM: central lymph node metastasis; LLNM: lateral lymph node metastasis.

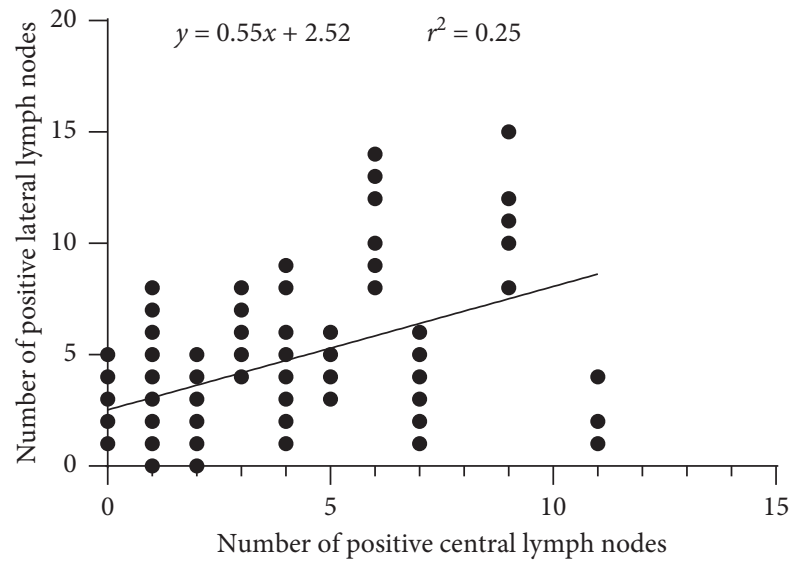

FIgURE 1: There is a positive correlation between the number of positive central and lateral lymph nodes.

reporting unsatisfactory results and roughly identifying the same risk factors, including male gender, younger age, the increase in tumor size, and so on $[10,13,31,36-38]$. In the present study, we also evaluated the predictive factors finding no new results. Currently, there are no prominent or particular indexes that could predict CLNM.

For PTC patients, therapeutic CLND is always necessary, while the controversy mainly arises around P-CLND. P-CLND has been considered as an overtreatment $[11,39]$. Several international guidelines [40-42] have suggested

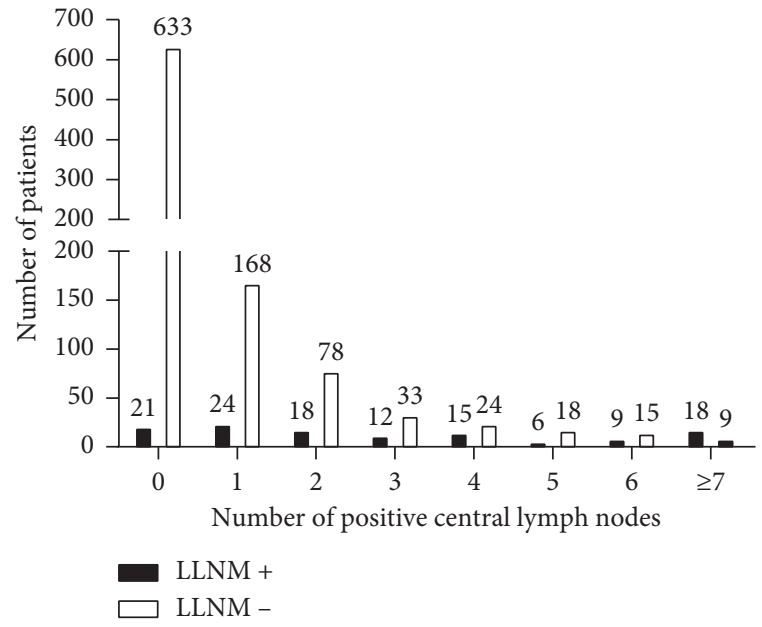

FIGURE 2: The incidence of lateral lymph node metastasis increased with the number of positive central lymph nodes. LLNM, lateral lymph node metastasis.

performing P-CLND only in patients with advanced primary tumors (T3 and T4), or if the information is to be used to plan further steps in therapy. However, the TNM staging system sometimes does not necessarily reflect less aggressive disease [43]. Therefore, there are inconsistent views on P-CLND. According to many clinicians, CLND should be considered even in PTC patients without lymphatic invasion $[31,44]$. These poor outcomes mainly occur in patients with 
LNM, especially CLNM [10]. Numerous studies have concluded that reoperation due to recurrence in cervical nodes is associated with an increase in postoperative complications, such as recurrent laryngeal nerve injury and hypoparathyroidism $[9,45]$. Reoperation has also been suggested as a risk factor for disease-specific mortality, which is a more serious problem [46]. Furthermore, a second operation is associated with a requirement for general anesthesia, patient stress, and higher medical healthcare cost [47].

In this study, all patients underwent P-CLND, but the rate of postoperative complications potentially caused by P-CLND was not higher than in other studies. As reported, the rate of persistent hypoparathyroidism ranges from $2.6 \%$ to $12 \%$ [31, 48-51], whereas in this study, 9 patients $(2.8 \%)$ undergoing total thyroidectomy were observed having persistent hypoparathyroidism. Meanwhile, the rate of transient hypoparathyroidism in this study (6\%) was lower than the reported data (ranging from $6.9 \%$ to $9.0 \%$ ) [31, 47]. Among 34 cases with vocal cord palsy, which implied recurrent laryngeal nerve injury, $22(2 \%)$ recovered within 1 to 6 months while the other $12(1.1 \%)$ patients had a persistent injury; while the rate of transient recurrent laryngeal nerve injury ranges from $1.1 \%$ to $6.0 \%$, the rate of persistent recurrent laryngeal nerve injury ranges from $0.2 \%$ to $3.6 \%[31,47-52]$. Several previous studies have reported that the incidence of chyle leakage ranges from $0.5 \%$ to $8.3 \%$ [53-55], whereas in this study, 5 patients (3.5\%) presented with chyle leakage and were cured within 20 days. Most of the patients recovered well. According to our results, routine P-CLND does not increase the risk of complications and can help to get a more accurate stage and to avoid the potential risk of the second operation. As a result, P-CLND during thyroid surgery appears as a reasonable management option.

There are some limitations to this study. First, it is a retrospective, single-institution study. Second, it lacks long-term follow-up results, so the data such as disease recurrence rates, postoperative radioiodine studies, thyroglobulin level fluctuation, and disease-free survival are still unknown. Third, the pathological subtypes were not distinguished in statistics, which might cause bias in the results. We will continue collecting clinicopathological data in the long-term follow-up as a consecutive report.

\section{Conclusion}

CLNM is frequently microscopic and not detectable preoperatively. Our results revealed that the probability of CLNM was closely related to the male gender, age $<55$, and the increase in tumor size. Those patients with an increase in tumor size and CLNM were extremely prone to LLNM. In addition, LLNM was more likely to happen in those with the more positive central lymph nodes. The rates of postoperative complications potentially caused by CLND were lower than in other studies. Routine P-CLND does not increase the risk of complications and should be considered as a reasonable surgical treatment for PTC.

\section{Data Availability}

The raw clinical data used to support the findings of this study are available from the corresponding author upon request.

\section{Conflicts of Interest}

The authors declare that they have no conflicts of interest.

\section{Authors' Contributions}

Yifan Chen and Shuo Chen contributed equally to this work.

\section{Acknowledgments}

This work was supported by the Startup Fund for Scientific Research of Fujian Medical University (Grant no. 2018QH1127).

\section{References}

[1] I. D. Hay, M. E. Hutchinson, T. Gonzalez-Losada et al., "Papillary thyroid microcarcinoma: a study of 900 cases observed in a 60-year period," Surgery, vol. 144, no. 6, pp. 980-988, 2008.

[2] Y. Ito, T. Uruno, K. Nakano et al., "An observation trial without surgical treatment in patients with papillary microcarcinoma of the thyroid," Thyroid, vol. 13, no. 4, pp. 381-387, 2003.

[3] I. Sugitani, K. Toda, K. Yamada, N. Yamamoto, M. Ikenaga, and Y. Fujimoto, "Three distinctly different kinds of papillary thyroid microcarcinoma should be recognized: our treatment strategies and outcomes," World Journal of Surgery, vol. 34, no. 6, pp. 1222-1231, 2010.

[4] C. I. Lundgren, P. Hall, P. W. Dickman, and J. Zedenius, "Clinically significant prognostic factors for differentiated thyroid carcinoma," Cancer, vol. 106, no. 3, pp. 524-531, 2006.

[5] D. Viola, G. Materazzi, L. Valerio et al., "Prophylactic central compartment lymph node dissection in papillary thyroid carcinoma: clinical implications derived from the first prospective randomized controlled single institution study," The Journal of Clinical Endocrinology \& Metabolism, vol. 100, no. 4, pp. 1316-1324, 2015.

[6] A. Goropoulos, K. Karamoshos, A. Christodoulou et al., "Value of the cervical compartments in the surgical treatment of papillary thyroid carcinoma," World Journal of Surgery, vol. 28, no. 12, pp. 1275-1281, 2004.

[7] J.-L. Roh, J.-M. Kim, and C. I. Park, "Lateral cervical lymph node metastases from papillary thyroid carcinoma: pattern of nodal metastases and optimal strategy for neck dissection," Annals of Surgical Oncology, vol. 15, no. 4, pp. 1177-1182, 2008.

[8] J. Y. Kwak, E.-K. Kim, M. J. Kim et al., "Papillary microcarcinoma of the thyroid: predicting factors of lateral neck node metastasis," Annals of Surgical Oncology, vol. 16, no. 5, pp. 1348-1355, 2009.

[9] R. Alvarado, M. S. Sywak, L. Delbridge, and S. B. Sidhu, "Central lymph node dissection as a secondary procedure for papillary thyroid cancer: is there added morbidity?" Surgery, vol. 145, no. 5, pp. 514-518, 2009.

[10] F. H. Liu, S. F. Kuo, C. Hsueh, T. C. Chao, and J. D. Lin, "Postoperative recurrence of papillary thyroid carcinoma with 
lymph node metastasis," Journal of Surgical Oncology, vol. 112, no. 2, pp. 149-154, 2015.

[11] N. Wada, Q.-Y. Duh, K. Sugino et al., "Lymph node metastasis from 259 papillary thyroid microcarcinomas," Annals of Surgery, vol. 237, no. 3, pp. 399-407, 2003.

[12] A. R. Shaha, "Editorial: complications of neck dissection for thyroid cancer," Annals of Surgical Oncology, vol. 15, no. 2, pp. 397-399, 2008.

[13] R.-C. Zeng, W. Zhang, E.-L. Gao et al., "Number of central lymph node metastasis for predicting lateral lymph node metastasis in papillary thyroid microcarcinoma," Head \& Neck, vol. 36, no. 1, pp. 101-106, 2014.

[14] Y.-S. Lim, J.-C. Lee, Y. S. Lee et al., "Lateral cervical lymph node metastases from papillary thyroid carcinoma: predictive factors of nodal metastasis," Surgery, vol. 150, no. 1, pp. 116-121, 2011.

[15] G.-Z. Xiao and L. Gao, "Central lymph node metastasis: is it a reliable indicator of lateral node involvement in papillary thyroid carcinoma?" World Journal of Surgery, vol. 34, no. 2, pp. 237-241, 2010.

[16] L. Davies, B. R. Roman, and L. G. Morris, "The thyroid cancer epidemic, 2017 perspective," Current Opinion in Endocrinology Diabetes \& Obesity, vol. 24, no. 5, p. 1, 2017.

[17] M. J. Schlumberger and M. Torlantano, "Papillary and follicular thyroid carcinoma," The New England Journal of Medicine, vol. 338, no. 4, pp. 297-306, 1998.

[18] A. Machens, C. Hofmann, S. Hauptmann, and H. Dralle, "Locoregional recurrence and death from medullary thyroid carcinoma in a contemporaneous series: 5-year results," European Journal of Endocrinology, vol. 157, no. 1, pp. 85-93, 2007.

[19] D. I. Kutler, A. D. Crummey, and W. I. Kuhel, "Routine central compartment lymph node dissection for patients with papillary thyroid carcinoma," Head \& Neck, vol. 34, no. 2, pp. 260-263, 2012.

[20] Y. Tao, C. Wang, L. Li et al., "Clinicopathological features for predicting central and lateral lymph node metastasis in papillary thyroid microcarcinoma: analysis of 66 cases that underwent central and lateral lymph node dissection," Molecular and Clinical Oncology, vol. 6, no. 1, pp. 49-55, 2017.

[21] C. Zhao, W. Jiang, Y. Gao, W. Niu, X. Zhang, and L. Xin, "Risk factors for lymph node metastasis (LNM) in patients with papillary thyroid microcarcinoma (PTMC): role of preoperative ultrasound," Journal of International Medical Research, vol. 45, no. 3, pp. 1221-1230, 2017.

[22] G. Sapuppo, F. Palermo, M. Russo et al., "Latero-cervical lymph node metastases (N1b) represent an additional risk factor for papillary thyroid cancer outcome," Journal of Endocrinological Investigation, vol. 40, no. Suppl 2, pp. 1-9, 2017.

[23] Z.-Z. Lu, Y. Zhang, S.-F. Wei et al., "Outcome of papillary thyroid microcarcinoma: study of 1,990 cases," Molecular and Clinical Oncology, vol. 3, no. 3, pp. 672-676, 2015.

[24] Q. Zhao, J. Ming, C. Liu et al., "Multifocality and total tumor diameter predict central neck lymph node metastases in papillary thyroid microcarcinoma," Annals of Surgical Oncology, vol. 20, no. 3, pp. 746-752, 2013.

[25] I. Vasileiadis, T. Karatzas, D. Vasileiadis et al., "Clinical and pathological characteristics of incidental and nonincidental papillary thyroid microcarcinoma in 339 patients," Head \& Neck, vol. 36, no. 4, pp. 564-570, 2014.

[26] C. Y. Gui, S. L. Qiu, Z. H. Peng, and M. Wang, "Clinical and pathologic predictors of central lymph node metastasis in papillary thyroid microcarcinoma: a retrospective cohort study," Journal of Endocrinological Investigation, vol. 41, no. 4, pp. 403-409, 2017.

[27] A. Machens, R. Hinze, O. Thomusch, and H. Dralle, "Pattern of nodal metastasis for primary and reoperative thyroid cancer," World Journal of Surgery, vol. 26, no. 1, pp. 22-28, 2002.

[28] A. Machens, H. J. Holzhausen, and H. Dralle, "Skip metastases in thyroid cancer leaping the central lymph node compartment," Archives of Surgery, vol. 139, no. 1, pp. 43-45, 2004.

[29] S. Grodski, L. Cornford, M. Sywak, S. Sidhu, and L. Delbridge, "Routine level VI lymph node dissection for papillary thyroid cancer: surgical technique," Anz Journal of Surgery, vol. 77, no. 4, pp. 203-208, 2007.

[30] X. Nie, Z. Tan, and M. Ge, "Skip metastasis in papillary thyroid carcinoma is difficult to predict in clinical practice," BMC Cancer, vol. 17, no. 1, pp. 1-8, 2017.

[31] M. Caliskan, J. H. Park, J. S. Jeong et al., "Role of prophylactic ipsilateral central compartment lymph node dissection in papillary thyroid microcarcinoma," Endocrine Journal, vol. 59, no. 4, pp. 305-311, 2012.

[32] L.-E. Tisell, B. Nilsson, J. Mölne et al., "Improved survival of patients with papillary thyroid cancer after surgical microdissection," World Journal of Surgery, vol. 20, no. 7, pp. 854-859, 1996.

[33] G. F. W. Scheumann, O. Gimm, G. Wegener, H. Hundeshagen, and H. Dralle, "Prognostic significance and surgical management of locoregional lymph node metastases in papillary thyroid cancer," World Journal of Surgery, vol. 18, no. 4, pp. 559-567, 1994.

[34] J. S. Choi, J. Kim, J. Y. Kwak, M. J. Kim, H. S. Chang, and E.-K. Kim, "Preoperative staging of papillary thyroid carcinoma: comparison of ultrasound imaging and CT," American Journal of Roentgenology, vol. 193, no. 3, pp. 871-878, 2009.

[35] M. Mulla and K.-M. Schulte, "Central cervical lymph node metastases in papillary thyroid cancer: a systematic review of imaging-guided and prophylactic removal of the central compartment," Clinical Endocrinology, vol. 76, no. 1, pp. 131-136, 2012.

[36] F. Cheng, Y. Chen, L. Zhu et al., "Risk factors for cervical lymph node metastasis of papillary thyroid microcarcinoma: a single-center retrospective study," International Journal of Endocrinology, vol. 2019, Article ID 8579828, 6 pages, 2019.

[37] Y. Luo, Y. Zhao, K. Chen et al., "Clinical analysis of cervical lymph node metastasis risk factors in patients with papillary thyroid microcarcinoma," Journal of Endocrinological Investigation, vol. 42, no. 2, pp. 227-236, 2019.

[38] P. Miccoli, M. N. Minuto, C. Ugolini et al., "Intrathyroidal differentiated thyroid carcinoma: tumor size-based surgical concepts," World Journal of Surgery, vol. 31, no. 5, pp. 888-894, 2007.

[39] A. R. Shaha, R. M. Tuttle, and J. P. Shah, "Papillary microcarcinoma of the thyroid," Journal of Surgical Oncology, vol. 95, no. 7, pp. 532-533, 2007.

[40] B. R. Haugen, E. K. Alexander, K. C. Bible et al., "American thyroid association management guidelines for adult patients with thyroid nodules and differentiated thyroid cancer: the American thyroid association guidelines task force on thyroid nodules and differentiated thyroid cancer," Thyroid, vol. 26, no. 1, pp. 1-133, 2015.

[41] R. I. Haddad, C. Nasr, L. Bischoff et al., "NCCN guidelines insights: thyroid carcinoma, version 2.2018," Journal of The National Comprehensive Cancer Network, vol. 16, no. 12, pp. 1429-1440, 2018. 
[42] F. Pacini, M. Schlumberger, H. Dralle et al., "European consensus for the management of patients with differentiated thyroid carcinoma of the follicular epithelium," European Journal of Endocrinology, vol. 154, no. 6, pp. 787-803, 2006.

[43] C. Casella, S. Ministrini, A. Galani, F. Mastriale, C. Cappelli, and P. Portolani, "The new TNM staging system for thyroid cancer and the risk of disease downstaging," Frontiers in Endocrinology, vol. 9, p. 541, 2018.

[44] S.-H. Lee, S.-S. Lee, S.-M. Jin, J.-H. Kim, and Y.-S. Rho, "Predictive factors for central compartment lymph node metastasis in thyroid papillary microcarcinoma," The Laryngoscope, vol. 118, no. 4, pp. 659-662, 2008.

[45] M. K. Kim, S. H. Mandel, Z. Baloch et al., "Morbidity following central compartment reoperation for recurrent or persistent thyroid cancer," Archives of Otolaryngology-Head \& Neck Surgery, vol. 130, no. 10, pp. 1214-1216, 2004.

[46] S. Young, A. Harari, S. Smooke-Praw, P. H. G. Ituarte, and M. W. Yeh, "Effect of reoperation on outcomes in papillary thyroid cancer," Surgery, vol. 154, no. 6, pp. 1354-1362, 2013.

[47] Y. S. Lee, S. W. Kim, S. W. Kim et al., "Extent of routine central lymph node dissection with small papillary thyroid carcinoma," World Journal of Surgery, vol. 31, no. 10, pp. 1954-1959, 2007.

[48] G. Mercante, A. Frasoldati, C. Pedroni et al., "Prognostic factors affecting neck lymph node recurrence and distant metastasis in papillary microcarcinoma of the thyroid: results of a study in 445 patients," Thyroid, vol. 19, no. 7, pp. 707-716, 2009.

[49] T. Zetoune, X. Keutgen, D. Buitrago et al., "Prophylactic central neck dissection and local recurrence in papillary thyroid cancer: a meta-analysis," Annals of Surgical Oncology, vol. 17, no. 12, pp. 3287-3293, 2010.

[50] M. Barczynski, A. Konturek, M. Stopa, and W. Nowak, "Prophylactic central neck dissection for papillary thyroid cancer," British Journal of Surgery, vol. 100, no. 3, pp. 410-418, 2013.

[51] W. Zhao, L. You, X. Hou et al., "The effect of prophylactic central neck dissection on locoregional recurrence in papillary thyroid cancer after total thyroidectomy: a systematic review and meta-analysis," Annals of Surgical Oncology, vol. 24, no. 8, pp. 2189-2198, 2017.

[52] A. Konturek and M. Barczyński, "The evolution and progress of mechanism and prevention of recurrent laryngeal nerve injury," Annals of Thyroid, vol. 3, p. 32, 2018.

[53] J.-L. Roh, D. H. Kim, and C. I. Park, "Prospective identification of chyle leakage in patients undergoing lateral neck dissection for metastatic thyroid cancer," Annals of Surgical Oncology, vol. 15, no. 2, pp. 424-429, 2008.

[54] G. Wu, X. Chang, Y. Xia, W. Huang, and W. M. Koch, "Prospective randomized trial of high versus low negative pressure suction in management of chyle fistula after neck dissection for metastatic thyroid carcinoma," Head \& Neck, vol. 34, no. 12, pp. 1711-1715, 2012.

[55] M. E. Kupferman, D. M. Patterson, S. J. Mandel, V. LiVolsi, and R. S. Weber, "Safety of modified radical neck dissection for differentiated thyroid carcinoma," The Laryngoscope, vol. 114, no. 3, pp. 403-406, 2004. 